\title{
Metabolic Dysfunction-Associated Fatty Liver Disease Better Predicts Incident Cardiovascular Disease
}

\author{
Seogsong Jeong ${ }^{1}$, Yun Hwan Oh², Seulggie Choi', Jooyoung Chang ${ }^{1}$, Sung Min Kim¹, Joung Sik Son ${ }^{3}$, Gyeongsil \\ $\mathrm{Lee}^{4}$, Won Kim ${ }^{5,6}$, and Sang Min Park ${ }^{1,4}$ \\ ${ }^{1}$ Department of Biomedical Sciences, Seoul National University Graduate School, Seoul, ${ }^{2}$ Department of Family Medicine, Jeju \\ National University School of Medicine, Jeju, ${ }^{3}$ Department of Family Medicine, Korea University Guro Hospital, ${ }^{4}$ Department of Family \\ Medicine, Seoul National University Hospital, ${ }^{5}$ Division of Gastroenterology and Hepatology, Department of Internal Medicine, Seoul \\ Metropolitan Government Seoul National University Boramae Medical Center, and ${ }^{6}$ Department of Internal Medicine, Seoul National \\ University College of Medicine, Seoul, Korea
}

See editorial on page 497.

\section{Article Info}

Received June 5, 2021

Revised July 17, 2021

Accepted August 3, 2021

Published online November 3, 2021

\section{Corresponding Author}

\section{Sang Min Park}

ORCID https://orcid.org/0000-0002-7498-4829

E-mail smpark.snuh@gmail.com

Won Kim

ORCID https://orcid.org/0000-0002-2926-1007

E-mail drwon1@snu.ac.kr
Background/Aims: Metabolic dysfunction (MD)-associated fatty liver disease is a new positive diagnostic criterion based on hepatic steatosis and MD. However, a comprehensive evaluation on the association of MD and hepatic steatosis with incident cardiovascular disease (CVD) has yet to be performed.

Methods: This retrospective cohort study included 333,389 participants from the Korean National Health Insurance Service database who received a health examination between 2009 and 2010. Hepatic steatosis was defined using the Korean National Health and Nutrition Examination Survey-derived nonalcoholic fatty liver disease scoring system. Cox proportional hazards regression was adopted to determine the adjusted hazard ratio (aHR) with 95\% confidence interval $(\mathrm{Cl})$ for CVD according to the presence of hepatic steatosis and MD, as well as the composite term.

Results: This study included 179,437 men and 153,952 women with a median age of 57 years. Hepatic steatosis with MD (aHR, 2.00; 95\% Cl, 1.89 to 2.13) and without MD (aHR, 1.30; 95\% $\mathrm{Cl}, 1.10$ to 1.54 ) significantly increased the risk of CVD compared to no steatosis without MD (reference). However, steatosis revealed no significant difference in the risk of CVD compared to no steatosis among participants with one MD (aHR, 1.09; 95\% Cl, 0.91 to 1.30). In participants with steatosis, the presence of one and $\geq 2 \mathrm{MDs}$ had aHR values of $1.25(95 \% \mathrm{Cl}, 0.87$ to 1.79$)$ and $1.71(95 \% \mathrm{Cl}, 1.22$ to 2.41$)$, respectively, compared to no MD.

Conclusions: Combined consideration of hepatic steatosis and MD was significantly associated with increased CVD risk and showed better predictive performance for CVD than hepatic steatosis or MD alone. (Gut Liver 2022;16:589-598)

Key Words: Fatty liver; Cardiovascular diseases; Cardiometabolic risk factors; Liver diseases

\section{INTRODUCTION}

Nonalcoholic fatty liver disease (NAFLD) was coined over 40 years ago by Ludwig et al. and defined as hepatic steatosis with the exclusion of other competing etiologies of chronic liver diseases. ${ }^{1,2}$ Despite the various preventive and therapeutic approaches, the prevalence of NAFLD continues to increase with significant impacts on clinical medicine and economic burden to the society that nearly one billion people are being affected. ${ }^{3}$ Considering grow- ing knowledges on the heterogeneity of the patients with NAFLD with respect to its major drivers, including sociodemographic, clinical, and biological characteristics, an international panel of experts has suggested metabolic dysfunction (MD)-associated fatty liver disease (MAFLD) as a more articulate term that leads the research community to the renewal of the nomenclature to accelerate the development of new therapeutic approaches. ${ }^{4}$

In response to unmet needs in this field, the diagnosis of MAFLD can now be made using positive criteria on the 
basis of hepatic steatosis irrespective of alcohol consumption and concomitant liver diseases, with three subphenotypes including (1) overweight or obesity, (2) lean or normal weight, and (3) type 2 diabetes mellitus (DM). Lean or normal weight MAFLD is diagnosed if at least two MDs coexist, which includes abnormalities in waist circumference (WC), blood pressure (BP), triglycerides (TG), high-density lipoprotein (HDL)-cholesterol, glucose levels, insulin resistance, and high-sensitivity C-reactive protein as proposed in the Adult Treatment Panel III. ${ }^{6,7}$ Currently, ultrasound, vibration-controlled transient elastography, computed tomography, magnetic resonance imaging and spectroscopy, and serum biomarker-based models with available data on the diagnostic and prognostic performances, such as fatty liver index (FLI), are recommended for the detection of hepatic steatosis. ${ }^{8,9}$

Recently, we have developed the Korean National Health and Nutrition Examination Survey-derived NAFLD scoring system (K-NAFLD) based on patient characteristics and serum biomarkers, which represents metabolic risk and insulin resistance. ${ }^{10}$ Given the close relationship between NAFLD, MD, and cardiovascular disease (CVD), there is an unmet need for updates on their associations with CVD. ${ }^{11,12}$ Herein, CVD risk was assessed according to the presence of hepatic steatosis, MD, and their composite term using a Korean nationwide cohort.

\section{MATERIALS AND METHODS}

\section{Study population}

The National Health Insurance Service (NHIS) of Korea provides mandatory health insurance for all Koreans with an enrollment of nearly $98 \%$ covering almost all types of healthcare services. ${ }^{13}$ The biannual health screening examinations of the NHIS include anthropometric measurements, laboratory examinations, medical history, prescriptions, and self-reported questionnaires for claims purposes. The Korean NHIS-National Health Screening Cohort is composed of approximately 500,000 participants aged between 40 and 79 years, which is about $10 \%$ of the total population. ${ }^{14}$ This study was conducted in accordance with the STROBE guidelines for cohort studies. The Institutional Review Board of Seoul National University Hospital approved the present study (IRB number: E-2002-0401099) in accordance with relevant guidelines and terms. The requirement for informed consent was waived as the NHIS data were anonymized according to strict confidentiality guidelines.

A total of 362,285 participants received a health examination between 2009 and 2010. Among them, 18,540 participants were excluded due to previous history of CVD before starting follow-up (index date, January 1, 2009) to avoid potential bias from recurrent CVD. In addition, participants with missing information, including liver function test $(\mathrm{n}=103)$, WC $(\mathrm{n}=133)$, body mass index (BMI; $\mathrm{n}=66)$, TG ( $\mathrm{n}=430)$, fasting serum glucose (FSG; $\mathrm{n}=4)$, total cholesterol $(n=41)$, HDL-cholesterol $(n=17), B P(n=30)$, physical activity $(\mathrm{n}=2,848)$, smoking $(\mathrm{n}=5,243)$, and alcohol consumption $(n=1,431)$, were excluded from the analysis. Finally, the study population consisted of 333,389 participants (Fig. 1).

\section{Definition of hepatic steatosis, NAFLD, and MAFLD}

The K-NAFLD score $(0.913 \times$ sex [2, if female; 1 , if male $]+0.089 \times \mathrm{WC}+0.032 \times[$ systolic $\mathrm{BP}+\mathrm{FSG}]+\mathrm{TG} \times 0.007+$ alanine aminotransferase $[$ ALT $] \times 0.105-20.929)$ was used in defining the hepatic steatosis. ${ }^{10}$ The K-NAFLD score of $<-3.285$ and $>0.884$ were defined as no hepatic steatosis and hepatic steatosis, respectively. NAFLD was considered present for participants with K-NAFLD score $>0.884$ without alcohol consumption and chronic viral hepatitis $B$ and $\mathrm{C}$, which were defined using the International Classification of Diseases 10th Revision (ICD-10) code of B18. ${ }^{15}$ MAFLD was defined as hepatic steatosis+BMI $\geq 23 \mathrm{~kg} / \mathrm{m}^{2}$ or FSG $\geq 126 \mathrm{mg} / \mathrm{dL}$ and/or prescription of antidiabetic drugs, or $\geq 2$ MDs; $W C \geq 90 \mathrm{~cm}$ for men and $\geq 80 \mathrm{~cm}$ for women, $\mathrm{BP} \geq 130 / 85 \mathrm{~mm} \mathrm{Hg}$ or specific drug treatment, TG $\geq 150 \mathrm{mg} / \mathrm{dL}$ or specific drug treatment, HDL cholesterol $<40 \mathrm{mg} / \mathrm{dL}$ for men and $<50 \mathrm{mg} / \mathrm{dL}$ for women, and prediabetes (FSG between 100 to $125 \mathrm{mg} / \mathrm{dL}$ ) were recognized as MDs. ${ }^{5}$

\section{Calculation of the FLI, LAP, and HSI}

The FLI was calculated by $\left(e^{0.953} \times \log _{e}[T G]+0.139 \times\right.$

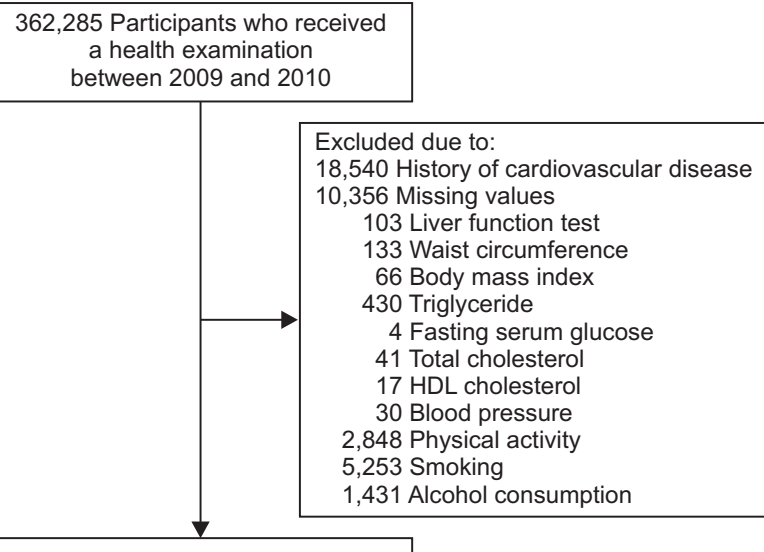

333,389 Study population

Fig. 1. Flowchart for the inclusion of study participants. 
$\mathrm{BMI}+0.718 \times \log _{\mathrm{e}}[\gamma$-glutamyl transpeptidase $]+0.053 \times$ $\mathrm{WC}-15.745) /\left(1+\mathrm{e}^{0.953} \times \log _{\mathrm{e}}[\mathrm{TG}]+0.139 \times \mathrm{BMI}+0.718 \times\right.$ $\log _{\mathrm{e}}[\gamma$-glutamyl transpeptidase $\left.]+0.053 \times \mathrm{WC}-15.745\right)$ $\times 100 .{ }^{16}$ Lipid accumulation product (LAP), an indicator for the severity of hepatic steatosis, was calculated by (WC-65) $\times$ TG for men and (WC-58) $\times$ TG for women, respectively. ${ }^{17}$ LAP was evaluated in dichotomous groups stratified by K-NAFLD score and hepatic steatosis index (HSI; $8 \times$ ALT-to-aspartate aminotransferase ratio+BMI [+2, if $\mathrm{DM} ;+2$ if female]) to support the association between the severity of hepatic steatosis and the K-NAFLD score (Supplementary Table 1). ${ }^{18}$

\section{Key variables}

The following covariates were considered key variables for adjustments in multivariate analyses: age, sex, insurance premium (upper half and lower half), BMI $\left(\mathrm{kg} / \mathrm{m}^{2}\right)$, FSG (mg/dL), total cholesterol (mg/dL), ALT (IU/L), smoking (never, previous, and current), alcohol consumption (never, 1-4 days/week, and $\geq 5$ days/week), physical activity (moderate-to-vigorous physical activity $\leq 1$ time/ week and $>1$ time/week), and the Charlson comorbidity index (CCI). The CCI was calculated in accordance with a previous study. ${ }^{19}$

\section{Incidence of CVD}

The attending physicians were required to insert the ICD-10 code for the primary disease for each patient upon hospitalization. CVD was operatively defined as 2 or more days of hospitalization due to coronary heart disease (ICD10 code: I20-I25) or stroke (ICD-10 code: I60-I69), which were adopted by the American Heart Association guidelines. ${ }^{20}$ The validity of the operational definition using the NHIS database was described elsewhere. ${ }^{21}$ Starting from January 1, 2009, all participants were followed for CVD and death, whichever occurred earlier, till December 31, 2015.

\section{Statistical analysis}

Continuous and categorical variables were presented with median (interquartile range; IQR) and frequency (\%), respectively. Between-group difference was evaluated using the chi-square test and the independent t-test for categorical and continuous variables, respectively. The incidence rate of CVD was evaluated by crude rate/1,000 personyear. The multivariate Cox proportional hazards regression model was applied for evaluation of adjusted hazard ratio (aHR) with 95\% confidence interval (CI) for CVD according to the hepatic steatosis. Considering potential bias due to other cause before the follow-up investigation, 1- and 2 -year of latent periods were washed out for sensitivity analyses. Subgroup analyses were conducted based on the key variables. For incident CVD in the overall follow-up period, receiver operating characteristic contrast estimation was carried out for NAFLD, hepatic steatosis, and MAFLD. A p-value of less than 0.05 was considered statistically significant. All data collection, mining, and statistical analyses were performed using SAS Enterprise Guide

Table 1. Baseline Characteristics of the Participants

\begin{tabular}{|c|c|}
\hline Characteristic & Participants (n=333,389) \\
\hline Age, yr & $57(51-64)$ \\
\hline \multicolumn{2}{|l|}{ Sex } \\
\hline Male & $179,437(53.8)$ \\
\hline Female & $153,952(46.2)$ \\
\hline \multicolumn{2}{|l|}{ Insurance premium* } \\
\hline Upper half & $216,989(65.1)$ \\
\hline Lower half & $116,400(34.9)$ \\
\hline Body mass index, $\mathrm{kg} / \mathrm{m}^{2}$ & $23.9(22.0-25.8)$ \\
\hline Waist circumference, $\mathrm{cm}$ & $82(76-87)$ \\
\hline Systolic blood pressure, $\mathrm{mm} \mathrm{Hg}$ & $124(115-134)$ \\
\hline Diastolic blood pressure, $\mathrm{mm} \mathrm{Hg}$ & 79 (70-83) \\
\hline Fasting serum glucose, $\mathrm{mg} / \mathrm{dL}$ & $96(88-106)$ \\
\hline Total cholesterol, mg/dL & 198 (175-224) \\
\hline Triglyceride, mg/dL & $117(83-169)$ \\
\hline HDL-cholesterol, mg/dL & $52(44-62)$ \\
\hline LDL-cholesterol, mg/dL & $117(96-140)$ \\
\hline Aspartate aminotransferase, IU/L & $24(20-29)$ \\
\hline Alanine aminotransferase, IU/L & $21(16-29)$ \\
\hline Y-Glutamyl transferase, IU/L & $24(16-40)$ \\
\hline \multicolumn{2}{|l|}{ Smoking } \\
\hline Never & $214,916(64.5)$ \\
\hline Past & $60,720(18.2)$ \\
\hline Current & 57,753 (17.3) \\
\hline \multicolumn{2}{|l|}{ Alcohol consumption } \\
\hline Never & 200,713 (60.2) \\
\hline $1-4$ day/wk & $116,074(34.8)$ \\
\hline$\geq 5$ day/wk & $16,602(5.0)$ \\
\hline \multicolumn{2}{|l|}{ Physical activity $^{\dagger}$} \\
\hline Inactive & $180,060(54.0)$ \\
\hline Active & $153,329(46.0)$ \\
\hline Chronic viral hepatitis & $7,884(2.4)$ \\
\hline Hypertension ${ }^{\ddagger}$ & 106,008 (31.8) \\
\hline Type 2 diabetes ${ }^{\S}$ & $32,148(9.6)$ \\
\hline Dyslipidemia" & $49,335(14.8)$ \\
\hline \multicolumn{2}{|l|}{ Charlson comorbidity index } \\
\hline 0 & $125,237(37.6)$ \\
\hline 1 & 108,838 (32.6) \\
\hline$\geq 2$ & 99,314 (29.8) \\
\hline
\end{tabular}

Data are presented as the median (interquartile range) or number (\%).

HDL, high-density lipoprotein; LDL, low-density lipoprotein.

*Proxy for socioeconomic status based on the National Health Insurance Service; ${ }^{\dagger}$ Active defined as moderate-to-vigorous physical activity $>1$ time/wk based on the self-report questionnaire; ${ }^{\ddagger}$ Defined as systolic blood pressure $\geq 140 \mathrm{~mm} \mathrm{Hg}$, diastolic blood pressure $\geq 90$ $\mathrm{mm} \mathrm{Hg}$ or prescription of antihypertensive drugs; ${ }^{\S}$ Defined as fasting serum glucose $\geq 126 \mathrm{mg} / \mathrm{dL}$ or prescription of antidiabetic drugs; "Defined as total cholesterol $\geq 240 \mathrm{mg} / \mathrm{dL}$ or prescription of antidyslipidemic drugs. 
7.1 (SAS Institute Inc., Cary, NC, USA).

\section{RESULTS}

\section{Subjects characteristics}

The descriptive characteristics of the study participants are shown in Table 1. There were 179,437 men (53.8\%) and 153,952 women $(46.2 \%)$ with a median age of 57 years (IQR, 51 to 64), a total follow-up period of 1,850,704 person-year, and a total CVD event of 32,411 cases. The median BMI and WC were $23.9 \mathrm{~kg} / \mathrm{m}^{2}$ (IQR, 22.0 to 25.8 ) and $82 \mathrm{~cm}$ (IQR, 76 to 87), respectively. Among entire study population, 46,319 participants (13.9\%) had hepatic steatosis. Compared to the participants with no hepatic steatosis $(n=287,070)$, the participants with hepatic steatosis $(n=46,319)$ revealed significantly higher proportion of men, smoking, alcohol consumption, chronic viral hepatitis, hypertension, type $2 \mathrm{DM}$, dyslipidemia, and $\mathrm{CCI} \geq 2$, indicating that the hepatic steatosis group had a higher cardiometabolic risk than the non-hepatic steatosis group
(Supplementary Table 2).

\section{Associations of hepatic steatosis, MD, and MAFLD with CVD risk}

We first examined the association of hepatic steatosis with CVD using the K-NAFLD and FLI (Supplementary Table 3). Hepatic steatosis was in direct proportion to CVD risk since both 4th quartile groups of K-NAFLD and FLI showed highly increased CVD risk compared to 1st quartile groups. The discriminative performance of the K-NAFLD in the prediction of CVD was more effective than that of the FLI in terms of the CVD incidence rates. Subsequently, the study population was stratified into four groups, including very low, low, intermediate, and high K-NAFLD. The median K-NAFLD of the participants with no ruled in nor ruled out for hepatic steatosis was -1.690 , which was set as the cutoff for stratification into low and intermediate KNAFLD groups. The incidence rate of CVD was lowest in very low K-NAFLD, followed by low, intermediate, and high K-NAFLD (Table 2). Among participants with no MD, the aHRs were 1.30 (95\% CI, 1.17 to 1.44$), 1.24$ (95\% CI,

Table 2. Association of Hepatic Steatosis with Incident Cardiovascular Disease According to the K-NAFLD Score and Presence of Metabolic Dysfunction

\begin{tabular}{|c|c|c|c|c|c|}
\hline & & $\mathrm{K}-\mathrm{NA}$ & score & & \\
\hline & Very low (no steatosis) & Low & Intermediate & High (steatosis) & 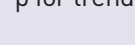 \\
\hline K-NAFLD score, range & $<-3.285$ & -3.285 to -1.690 & -1.690 to 0.884 & $>0.884$ & \\
\hline Overall population & & & & & \\
\hline No. & 75,806 & 105,611 & 105,653 & 46,319 & \\
\hline Event (\%) & $5,131(6.8)$ & $9,740(9.2)$ & $11,961(11.3)$ & $5,579(12.0)$ & \\
\hline PY & 428,536 & 588,447 & 580,908 & 252,813 & \\
\hline Crude rate/1,000 PY & 12.0 & 16.6 & 20.6 & 22.1 & \\
\hline $\operatorname{aHR}(95 \% \mathrm{Cl})$ & 1.00 (reference) & $1.22(1.18-1.27)^{+}$ & $1.42(1.36-1.48)^{+}$ & $1.63(1.55-1.73)^{+}$ & $<0.001$ \\
\hline No metabolic dysfunctio & & & & & \\
\hline No. & 35,854 & 14,109 & 3,143 & 767 & \\
\hline Event $(\%)$ & $1,493(4.2)$ & $811(5.7)$ & $176(5.6)$ & $39(5.1)$ & \\
\hline PY & 205,718 & 79,909 & 17,748 & 4,355 & \\
\hline Crude rate/1,000 PY & 7.3 & 10.1 & 9.9 & 9.0 & \\
\hline $\operatorname{aHR}(95 \% \mathrm{CI})$ & 1.00 (reference) & $1.30(1.17-1.44)^{+}$ & $1.24(1.01-1.52)^{*}$ & $1.27(0.78-2.06)$ & $<0.001$ \\
\hline Metabolic dysfunction=1 & & & & & \\
\hline No. & 29,963 & 40,648 & 17,246 & 3,637 & \\
\hline Event $(\%)$ & $2,406[8.0]$ & $3,181(7.8)$ & 1,432 (8.3) & 301 (8.3) & \\
\hline PY & 168,295 & 228,335 & 96,312 & 20,220 & \\
\hline Crude rate/1,000 PY & 14.3 & 13.9 & 14.9 & 14.9 & \\
\hline $\operatorname{aHR}(95 \% \mathrm{CI})$ & 1.00 (reference) & $1.01(0.95-1.08)$ & $1.10(1.01-1.20)^{*}$ & $1.09(0.91-1.30)$ & 0.111 \\
\hline Metabolic dysfunction $\geq 2$ & & & & & \\
\hline No. & 9,989 & 50,854 & 85,264 & 41,915 & \\
\hline Event $(\%)$ & $1,232(12.3)$ & $5,748(11.3)$ & $10,353(12.1)$ & $5,239(12.5)$ & \\
\hline PY & 54,524 & 280,203 & 466,848 & 228,239 & \\
\hline Crude rate/1,000 PY & 22.6 & 20.5 & 22.2 & 23.0 & \\
\hline $\operatorname{aHR}(95 \% \mathrm{CI})$ & 1.00 (reference) & $0.96(0.90-1.03)$ & $1.05(0.98-1.13)$ & $1.17(1.08-1.27)^{+}$ & $<0.001$ \\
\hline
\end{tabular}

aHR was calculated using the Cox proportional hazards model after adjustments for age, sex, insurance premium, body mass index, alanine aminotransferase, smoking, alcohol consumption, physical activity, and Charlson comorbidity index.

K-NAFLD, Korean National Health and Nutrition Examination Survey-derived nonalcoholic fatty liver disease; PY, person-year; aHR, adjusted hazard ratio; $\mathrm{Cl}$, confidence interval.

${ }^{*} p<0.05,{ }^{\dagger} p<0.001$. 
1.01 to 1.52 ), and 1.27 ( $95 \% \mathrm{CI}, 0.78$ to 2.06 ) for low, intermediate, and high K-NAFLD, respectively. In addition, Hepatic steatosis was found to significantly increase CVD risk (aHR, 1.17; 95\% CI, 1.08 to 1.27) only among participants with $\geq 2$ MDs, who corresponded to the MAFLD group, suggesting that significantly increased CVD risk in overall population might be highly attributed to participants with $\geq 2$ MDs. Subgroup analysis of the effect of hepatic steatosis on incident CVD among subjects with MD showed that a significant trend of increased CVD risk by hepatic steatosis severity was observed in all subgroups except for $\mathrm{CCI}=0$ (Supplementary Table 4). To confirm whether the prognostic impact of MAFLD on incident CVD is consistent across various cardiovascular risk factors, subgroup analyses were carried out after stratifying the study population into MAFLD and non-MAFLD (Fig. 2). MAFLD significantly increased CVD risk only in the age $\geq 65$ years, male, past or current smoker, BMI $<25 \mathrm{~kg} / \mathrm{m}^{2}$, and CCI $\geq 1$ subgroups.

\begin{tabular}{|c|c|c|c|c|c|c|}
\hline \multirow[b]{2}{*}{ Subgroup } & \multicolumn{3}{|c|}{ No MAFLD } & \multicolumn{3}{|c|}{ MAFLD } \\
\hline & No. & Events & $\begin{array}{c}\text { Event/ } \\
1,000 \mathrm{PY}\end{array}$ & No. & Events & $\begin{array}{c}\text { Event/ } \\
\text { 1,000 PY }\end{array}$ \\
\hline \multicolumn{7}{|l|}{ Age } \\
\hline$<65 \mathrm{yr}$ & 218,905 & 14,659 & 11.9 & 35,070 & 3,381 & 17.5 \\
\hline$\geq 65 \mathrm{yr}$ & 69,291 & 12,246 & 32.7 & 10,123 & 2,125 & 39.7 \\
\hline \multicolumn{7}{|l|}{ Sex } \\
\hline Male & 149,597 & 14,758 & 17.9 & 29,840 & 3,519 & 21.7 \\
\hline Female & 138,599 & 12,147 & 15.6 & 15,353 & 1,987 & 23.6 \\
\hline \multicolumn{7}{|l|}{ Cigarette smoking } \\
\hline Never smoker & 190,230 & 17,295 & 16.2 & 24,686 & 3,102 & 22.9 \\
\hline Past/current smoker & 97,966 & 9,610 & 17.8 & 20,507 & 2,404 & 21.6 \\
\hline \multicolumn{7}{|l|}{ Body mass index } \\
\hline$<25.0 \mathrm{~kg} / \mathrm{m}^{2}$ & 203,228 & 17,874 & 15.7 & 15,769 & 2,015 & 23.4 \\
\hline$\geq 25.0 \mathrm{~kg} / \mathrm{m}^{2}$ & 84,968 & 9,031 & 19.3 & 29,424 & 3,491 & 21.8 \\
\hline \multicolumn{7}{|l|}{ Physical activity } \\
\hline Inactive & 155,252 & 15,666 & 18.1 & 24,808 & 3,232 & 23.9 \\
\hline Active & 132,944 & 11,239 & 15.2 & 20,385 & 2,274 & 20.4 \\
\hline \multicolumn{7}{|l|}{ Chronic viral hepatitis } \\
\hline Present & 6,431 & 625 & 17.6 & 1,453 & 168 & 21.2 \\
\hline Absent & 281,765 & 26,280 & 16.8 & 43,740 & 5,338 & 22.4 \\
\hline \multicolumn{7}{|c|}{ Charlson comorbidity index } \\
\hline 0 & 111,973 & 5,590 & 8.7 & 13,264 & 816 & 10.9 \\
\hline$\geq 1$ & 176,223 & 21,315 & 22.1 & 31,929 & 4,690 & 27.3 \\
\hline
\end{tabular}
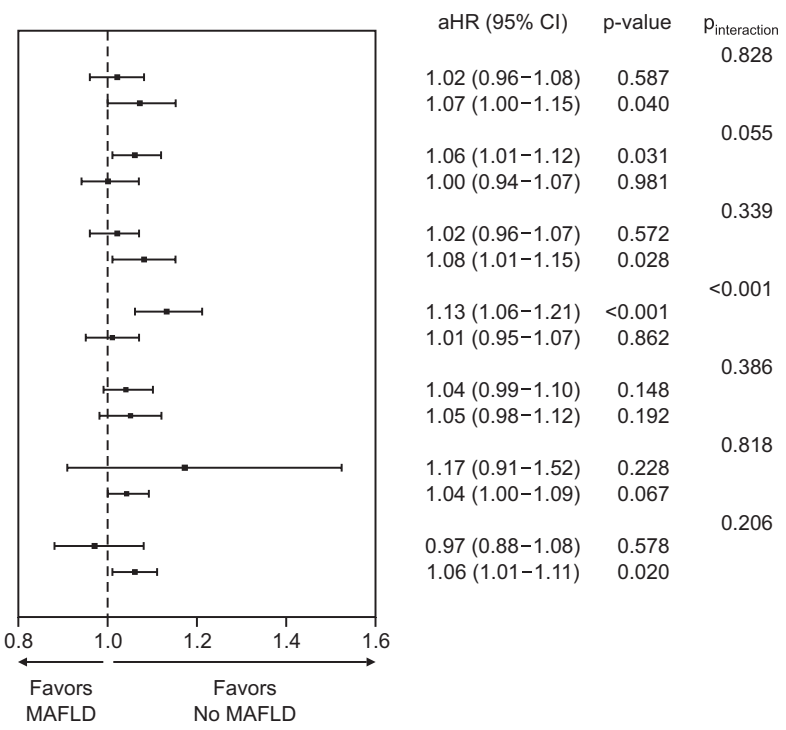

Fig. 2. Subgroup analysis of the effect of MAFLD on incident cardiovascular disease. aHR was calculated using the Cox proportional hazards model after adjustments for age, sex, insurance premium, body mass index, systolic blood pressure, fasting serum glucose, total cholesterol, alanine aminotransferase, smoking, alcohol consumption, physical activity, and Charlson comorbidity index. MAFLD was defined using the Korean National Health and Nutrition Survey nonalcoholic fatty liver disease score.

MAFLD, metabolic dysfunction-associated fatty liver disease; PY, person-year; aHR, adjusted hazard ratio; Cl, confidence interval.

Table 3. Associations of Hepatic Steatosis and MAFLD with Incident Cardiovascular Disease

\begin{tabular}{|c|c|c|c|c|c|}
\hline & No. & Event $(\%)$ & PY & $\begin{array}{c}\text { Crude rate/ } \\
1,000 \mathrm{PY}\end{array}$ & $\operatorname{aHR}(95 \% \mathrm{Cl})$ \\
\hline \multicolumn{6}{|l|}{ K-NAFLD score* } \\
\hline Very low* & 48,012 & $3,419(7.1)$ & 171,111 & 20.0 & 1.00 (reference) \\
\hline Low-intermediate $^{\dagger}$ & 124,658 & 14,126 (11.3) & 687,398 & 20.5 & $1.30(1.24-1.35)^{\S}$ \\
\hline $\mathrm{High}^{\ddagger}$ & 23,190 & $3,169(13.7)$ & 125,952 & 25.2 & $1.55(1.44-1.65)^{\S}$ \\
\hline \multicolumn{6}{|l|}{ Hepatic steatosis } \\
\hline Very low* & 75,806 & $5,131(6.8)$ & 428,536 & 12.0 & 1.00 (reference) \\
\hline Low-intermediate $^{\dagger}$ & 211,264 & $21,701(10.3)$ & $1,169,356$ & 18.6 & $1.30(1.25-1.34)^{\S}$ \\
\hline $\mathrm{High}^{\ddagger}$ & 46,319 & $5,579(12.0)$ & 252,813 & 22.1 & $1.55(1.47-1.63)^{\S}$ \\
\hline $\mathrm{MD}=0$ & 767 & $39(5.1)$ & 4,355 & 9.0 & 1.00 (reference) \\
\hline$M D=1$ & 3,637 & 301 (8.3) & 20,220 & 14.9 & 1.25 (0.87-1.79) \\
\hline$M D \geq 2$ & 41,915 & $5,239(12.5)$ & 228,239 & 23.0 & $1.71(1.22-2.41)^{\S}$ \\
\hline
\end{tabular}

aHR was calculated using the Cox proportional hazards model after adjustments for age, sex, insurance premium, body mass index, alanine aminotransferase, smoking, alcohol consumption, physical activity, and Charlson comorbidity index. NAFLD and hepatic steatosis were defined using the K-NAFLD score.

MAFLD, metabolic dysfunction-associated fatty liver disease; PY, person-year; aHR, adjusted hazard ratio; Cl, confidence interval; NAFLD, nonalcoholic fatty liver disease; K-NAFLD, Korean National Health and Nutrition Examination Survey-derived nonalcoholic fatty liver disease; MD, metabolic dysfunction.

${ }^{*}<-3.285$, NAFLD-ruled out; ${ }^{\dagger}-3.285$ to $0.884 ;{ }^{\ddagger}>0.884$, NAFLD-ruled in; ${ }^{\S} \mathrm{p}<0.001$. 
There was a significant interaction between BMI and MAFLD, which may be associated with the high incidence rate of CVD in participants with no MAFLD and BMI $\geq 25$ $\mathrm{kg} / \mathrm{m}^{2}$. There were no other significant interactions with MAFLD.

When examining the combined effect of the K-NAFLD and MD on CVD risk, both the K-NAFLD and MD additively increased the risk of CVD (Fig. 3). aHR of the K-NAFLD $>0.884$ and $\geq 2$ MDs group (i.e., the MAFLD group), was 2.22 ( $95 \% \mathrm{CI}, 2.07$ to 2.38 ) compared to the K-NAFLD $<-3.285$ and no MD group (i.e., the reference group) (Supplementary Table 5). Considering the validity of the FLI in the reflection of hepatic steatosis, we stratified the participants according to the IQR of the FLI. In accordance with the K-NAFLD, the 4th quartile of the FLI with $\geq 2$ MDs had the highest aHR of 2.23.

\section{Sensitivity analyses on the associations of hepatic steatosis and MAFLD with CVD risk}

To support the aforementioned effects of hepatic steatosis and MAFLD on incident CVD, 1 to 2 years of latent periods were washed out to exclude potentially uncontrolled factors. After 1-year wash out, hepatic steatosis lost its significant association with the risk of incident CVD, whereas the association of MAFLD with incident CVD risk remained significant (aHR, 1.05; 95\% CI, 1.00 to 1.10) (Supplementary Table 6). After washing out 2 years of latent period, similar results were found for hepatic steatosis and MAFLD, supporting that MAFLD is an independent predictor of incident CVD. We also adopted the HSI as a proxy for hepatic steatosis and NAFLD and reaffirmed that MAFLD was significantly associated with increased CVD risk (aHR, 1.15; 95\% CI, 1.11 to 1.19) in agreement with the findings based on the K-NAFLD and FLI (Supplementary Table 7).

Table 4. Receiver Operating Characteristic Contrast Estimation and Area Under the Curve Values of NAFLD, Hepatic Steatosis, and MAFLD for Incident Cardiovascular Disease

\begin{tabular}{lcccc}
\hline & AUC & $95 \% \mathrm{Cl}$ & Estimate (95\% CI) & $\mathrm{p}$-value \\
\hline NAFLD & 0.722 & $0.719-0.724$ & Reference & \\
Hepatic steatosis & 0.722 & $0.719-0.724$ & $4.6 \mathrm{E}-6(-1.0 \mathrm{E}-5$ to 2.0E-5) & 0.547 \\
MAFLD & 0.722 & $0.719-0.724$ & $9.3 \mathrm{E}-6(-3.8 \mathrm{E}-7$ to 2.0E-5) & 0.060 \\
\hline
\end{tabular}

Receiver operating characteristic curves were drawn for overall incident cardiovascular disease by logistic regression after adjustments for age, sex, insurance premium, body mass index, systolic blood pressure, fasting serum glucose, total cholesterol, alanine aminotransferase, smoking, alcohol consumption, physical activity, and Charlson comorbidity index. NAFLD, hepatic steatosis, and MAFLD were defined using the Korean National Health and Nutrition Survey NAFLD score.

NAFLD, nonalcoholic fatty liver disease; MAFLD, metabolic dysfunction-associated fatty liver disease; AUC, area under the curve; Cl, confidence interval.

Table 5. Associations of MAFLD Subphenotypes with Incident Cardiovascular Disease

\begin{tabular}{|c|c|c|c|c|c|c|}
\hline & \multicolumn{3}{|c|}{ No MAFLD } & \multicolumn{3}{|c|}{ MAFLD } \\
\hline & \multicolumn{3}{|c|}{ K-NAFLD score } & \multicolumn{3}{|c|}{ K-NAFLD score, high (>0.884) } \\
\hline & $\begin{array}{l}\text { Very low } \\
<-3.285\end{array}$ & $\begin{array}{c}\text { Low } \\
-3.285 \text { to }-1.690\end{array}$ & $\begin{array}{l}\text { Intermediate } \\
-1.690 \text { to } 0.884\end{array}$ & $\begin{array}{l}\text { DM-negative over- } \\
\text { weight/obese* }\end{array}$ & $\begin{array}{c}\text { DM-negative } \\
\text { normal weight }^{\dagger}\end{array}$ & $\mathrm{DM}^{\ddagger}$ \\
\hline Event (\%) & $5,131(6.8)$ & $9,740(9.2)$ & $11,961(11.3)$ & 2,592 (7.3) & 333 (11.5) & $2,314(15.1)$ \\
\hline PY & 428,536 & 588,447 & 580,908 & 131,445 & 14,564 & 82,229 \\
\hline Crude rate/1,000 PY & 12.0 & 16.6 & 20.6 & 19.7 & 22.9 & 28.5 \\
\hline $\operatorname{HR}(95 \% \mathrm{Cl})^{\S}$ & 1.00 (reference) & $1.38(1.33-1.44)^{\ddagger \ddagger}$ & $1.72(1.66-1.78)^{\ddagger \ddagger}$ & $1.67(1.58-1.75)^{\ddagger \ddagger}$ & $1.76(1.55-1.99)^{\ddagger \neq}$ & $2.44(2.32-2.57)^{\ddagger \ddagger}$ \\
\hline $\operatorname{aHR}(95 \% \mathrm{CI})^{\|}$ & 1.00 (reference) & $1.22(1.18-1.27)^{\ddagger \ddagger}$ & $1.46(1.40-1.52)^{\ddagger \neq}$ & $1.60(1.50-1.71)^{\ddagger \ddagger}$ & $1.68(1.47-1.91)^{\ddagger \neq}$ & $2.11(1.99-2.25)^{\ddagger \neq}$ \\
\hline $\operatorname{aHR}(95 \% \mathrm{CI})^{\pi}$ & 1.00 (reference) & $1.22(1.18-1.27)^{\ddagger \ddagger}$ & $1.42(1.36-1.47)^{\ddagger \ddagger}$ & $1.55(1.45-1.65)^{\ddagger \ddagger}$ & $1.55(1.36-1.77)^{\ddagger \ddagger}$ & $1.78(1.67-1.89)^{\ddagger \neq}$ \\
\hline aHR $(95 \% \mathrm{CI})^{\#}$ & 1.00 (reference) & $1.05(1.00-1.09)^{* *}$ & $1.07(1.02-1.12)^{\dagger+}$ & $1.02(0.95-1.10)$ & $1.03(0.90-1.18)$ & $1.18(1.10-1.26)^{\ddagger \neq}$ \\
\hline
\end{tabular}

HR was calculated using the Cox proportional hazards model.

MAFLD, metabolic dysfunction-associated fatty liver disease; K-NAFLD, Korean National Health and Nutrition Examination Survey-derived nonalcoholic fatty liver disease; DM, diabetes mellitus; PY, person-year; HR, hazard ratio; aHR, adjusted HR; $\mathrm{Cl}$, confidence interval.

${ }^{*}$ Body mass index $(B M I) \geq 23 \mathrm{~kg} / \mathrm{m}^{2} ;{ }^{\dagger} \mathrm{BMI}<23 \mathrm{~kg} / \mathrm{m}^{2}$; ${ }^{\dagger}$ Defined as fasting serum glucose $\geq 126 \mathrm{mg} / \mathrm{dL}$ or prescription of antidiabetic drugs; ${ }^{\S} U$ nadjusted; "Adjusted for age, sex, insurance premium, BMI, and alanine aminotransferase; "Adjusted for factors included in model B plus smoking,

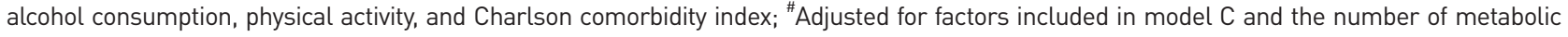
dysfunctions. MAFLD was defined using the Korean National Health and Nutrition Survey nonalcoholic fatty liver disease hepatic steatosis score; ${ }^{* *} \mathrm{p}<0.05 ;{ }^{+\dagger} \mathrm{p}<0.01 ;{ }^{\text {} \neq} \mathrm{p}<0.001$. 

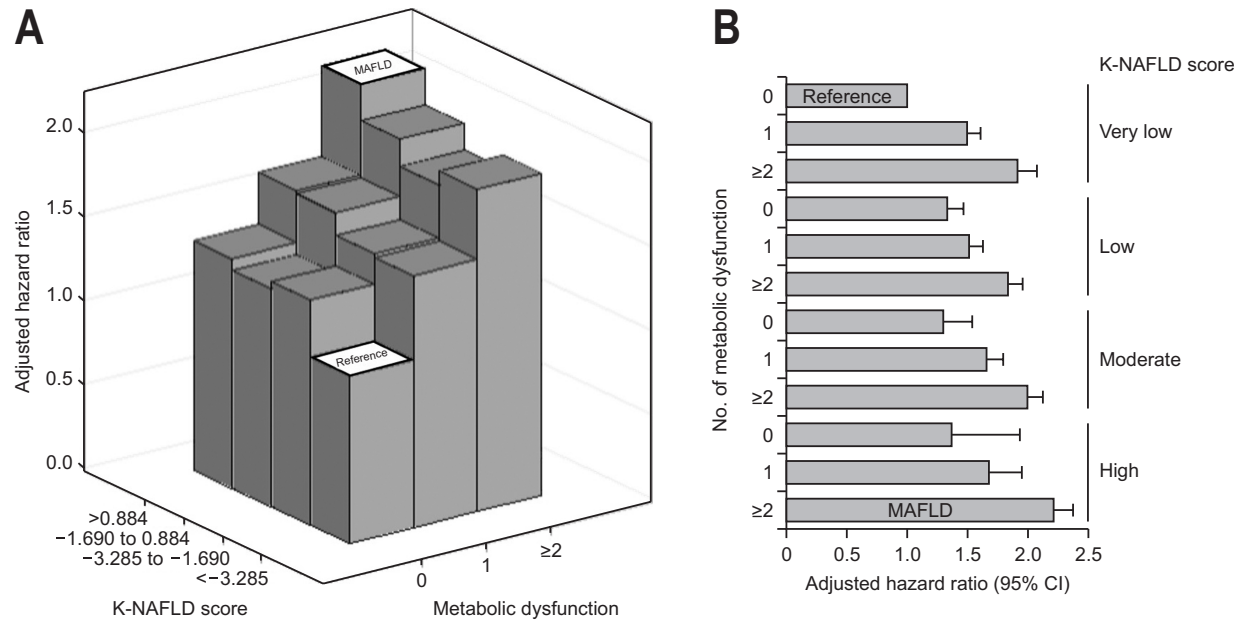

Fig. 3. Joint effect of hepatic steatosis and metabolic dysfunction on incident cardiovascular disease. aHR was calculated using the Cox proportional hazards model after adjustments for age, sex, insurance premium, body mass index, alanine aminotransferase, smoking, alcohol consumption, physical activity, and Charlson comorbidity index. (A) Three-dimensional plotting of the composite effect of the K-NAFLD score and the number of metabolic dysfunctions on the risk of cardiovascular disease. (B) Two-dimensional plotting of the composite effect of the K-NAFLD score and the number of metabolic dysfunctions on the risk of cardiovascular disease.

MAFLD, metabolic dysfunction-associated fatty liver disease; K-NAFLD, Korean National Health and Nutrition Examination Survey-derived nonalcoholic fatty liver disease; $\mathrm{Cl}$, confidence interval.

\section{Comparison of NAFLD and hepatic steatosis with and without MD in the prediction of CVD risk}

As for the evaluation on association of NAFLD with CVD, participants with hepatitis virus infection and/or alcohol consumption were excluded $(n=137,529)$, and the remaining 195,860 participants were divided into very low ( $\mathrm{n}=48,012$; NAFLD-ruled out), low-intermediate $(\mathrm{n}=124,658)$, and high $(\mathrm{n}=23,190$; NAFLD-ruled in) KNAFLD score groups (Table 3). The incidence rates of CVD were 20.0, 20.5, and 25.2 in the very low, low-to-intermediate, and high K-NAFLD score groups, respectively. In terms of hepatic steatosis that included participants with hepatitis virus infection and/or alcohol consumption, the incidence rates of CVD were 12.0, 18.6, and 22.1 in very low, low-to-intermediate, and high K-NAFLD score groups, respectively. Furthermore, stratification of the participants with hepatic steatosis into $\mathrm{MD}=0,=1$, and $\geq 2$ (MAFLD; $n=41,915$ ) revealed that the no MD group had the lowest incidence rate (9.0) despite the presence of hepatic steatosis. Moreover, MAFLD was more predictive of overall CVD incidence in the receiver operating characteristic contrast estimation compared to NAFLD without statistical significance $(\mathrm{p}=0.060)$ (Table 4$)$.

\section{Associations of the different MAFLD subphenotypes with CVD risk}

When stratifying MAFLD into three subphenotypes, DM-negative overweight/obese, DM-negative normal weight, and DM-positive subphenotypes showed significantly increased CVD risk in all unadjusted and adjusted models (Table 5). However, after further adjusting for the number of MD, only DM-MAFLD subtype significantly showed an increased risk of CVD despite the attenuation of statistical significance (aHR, 1.18; 95\% CI, 1.10 to 1.26), but both DM-negative overweight/obese and DMnegative normal weight subphenotypes lost their statistical significance. As for the other components of the metabolic dysfunction, WC-abnormal MAFLD was found to have comparative CVD risk while $\mathrm{BP}$, TG, and HDL cholesterolabnormal groups revealed higher CVD risk compared to no MAFLD group (Supplementary Table 8).

\section{DISCUSSION}

Recently, an international consensus of experts proposed MAFLD as new clearer nomenclature with positive diagnostic criteria to surmount the limitations on an operational definition of NAFLD. ${ }^{5,22}$ In the current study, both hepatic steatosis and MD were unfavorable prognosticators of incident CVD. In particular, the composite term, MAFLD conferred the most highly elevated risk of CVD in general population. The incidence rate of CVD was lowest in the very low K-NAFLD score and no MD group, whereas it was highest in the high K-NAFLD score and MD $\geq 2$ group. These results indicate the better performance of MAFLD in the prediction of CVD risk, circumventing the complicated use of both hepatic steatosis and MD.

The major difference between NAFLD and MAFLD is that MAFLD is a set of new positive criteria for the diag- 
nosis, regardless of other concomitant liver diseases and alcohol consumption. In addition, the change from NAFLD to MAFLD criteria is suggested to identify larger number of participants with metabolically complicated fatty liver disease who are at increased CVD risk. ${ }^{23}$ A previous study demonstrated that MD does not independently increase CVD risk among U.S. population with NAFLD. ${ }^{24}$ A recent study by Lee et al. ${ }^{25}$ has found that both NAFLD only and MAFLD only groups are associated with higher CVD risk after adjustment for household income, residential area, CCI, smoking, exercise, and estimated glomerular filtration rate. The aHR was higher in MAFLD only group (1.43) compared to NAFLD only group (1.09) and neither fatty liver disease group (reference). This may be associated with MDs as identified in this study. The major difference between their study and the present study is the specific evaluation of hepatic steatosis, which was stratified into very low (ruled out), low, intermediate, and high (ruled in) K-NAFLD groups. Accordingly, we could newly identify that very low hepatic steatosis status (ruled out) is associated with lower CVD risk compared to the others among participants with no MD. In addition, high hepatic steatosis status further increased CVD risk compared to very low hepatic steatosis status even among participants with $\geq 2$ MDs. Furthermore, CVD risk varied among different MAFLD subphenotypes. Specifically, nondiabetic MAFLD subphenotypes showed no significant associations with CVD risk, whereas diabetic MAFLD subphenotype demonstrated significantly increased risk of CVD compared to the subjects without hepatic steatosis, suggesting that $\mathrm{MD}$ may be an independent predictor of incident CVD in nondiabetic participants with hepatic steatosis.

Despite growing evidence on the association of either NAFLD or MD with CVD, it may be difficult to evaluate the prognostic impact of hepatic and metabolic risk factors on CVD risk in a unified and precise manner. In addition, conflicting results exist regarding the prognostic impact of NAFLD on the risk of CVD, as confirmed by the metaanalysis of 16 observational studies. ${ }^{26}$ Recently, we developed the K-NAFLD score and confirmed its significant associations with metabolic risks and insulin resistance. ${ }^{10}$ This scoring system allows for an operational definition of NAFLD and its application in the nationwide populationbased cohort. Furthermore, CVD risk in subjects with hepatic steatosis should be analyzed separately in different subgroups with varying degree of metabolic risk despite clear evidence on the link between NAFLD and cardiometabolic risk. In the present study, higher K-NAFLD was associated with increased CVD risk in overall population. Among participants with no metabolic dysfunction, participants with very low K-NAFLD score showed lower
CVD risk. In contrast, high K-NAFLD score was associated with increased CVD risk among participants with $\geq 2$ metabolic dysfunctions. These results suggest that evaluating hepatic steatosis allows better estimation of CVD risk, especially among participants with similar MD status. With an emerging concept of MAFLD, metabolic risk and hepatic steatosis seem to be now evaluable precisely in a concurrent manner.

In this study, the K-NAFLD which was also found to be associated with the severity of hepatic steatosis, was in direct proportion to the risk of CVD even after adjustments for sociodemographic characteristics (age, sex, and insurance premium), anthropometric measurements (BMI and WC), serologic characteristics (FSG, total cholesterol, and ALT), lifestyle behaviors (smoking, alcohol consumption, and physical activity), and the CCI, suggesting that the K-NAFLD may be an independent predictor of incident CVD. Even participants with low K-NAFLD had significantly increased CVD risk compared to those with very low K-NAFLD. In contrast, no significant increase in CVD risk was observed in participants with one MD compared to no $\mathrm{MD}$ among participants with hepatic steatosis, which may reinforce the international consensus on the definition of MAFLD as hepatic steatosis with $\geq 2$ MDs. However, it should be validated in future large-scale confirmatory studies.

From our perspective, better performance of MAFLD versus NAFLD in the prediction of CVD risk might be attributed to the inclusion of participants with alcohol consumption and chronic viral hepatitis in the MAFLD group. Moreover, our results indicated that participants with hepatic steatosis had $3.0 \%$ and $1.0 \%$ higher proportion of alcohol consumption $\geq 5$ days/week and chronic viral hepatitis, respectively, than those without hepatic steatosis. Repeated binge drinking or excessive cumulative alcohol consumption has been confirmed to have deleterious cardiovascular health consequences by increasing the incidence of CVD and related mortality. ${ }^{27,28}$ Moreover, chronic viral hepatitis, including hepatitis $\mathrm{B}$ and $\mathrm{C}$, was found to be associated with increased CVD risk. ${ }^{29,30}$ However, considering scarce evidence in literature and a previous metaanalysis on the impact of hepatitis B virus infection on coronary heart disease risk, it remains unclear whether hepatitis B virus infection increases CVD risk. ${ }^{31}$

Although our findings confirmed the deleterious effects of hepatic steatosis and MD on CVD risk, the current study had several inherent limitations that need to be considered when interpreting the results. First, the homeostasis model assessment for insulin resistance and high-sensitivity Creactive protein level, which are integral components for the diagnostic criteria of MAFLD, were not available in 
the NHIS database. Second, the presence and severity of hepatic steatosis were defined using the K-NAFLD, FLI, HSI, and LAP due to the nature of a large-scale cohort in which radiological and histological data were not available. Therefore, further prospective studies are warranted to validate our results using more accurate assessment tools of hepatic steatosis, such as imaging or biopsy. Finally, the current study adopted the ethnicity-specific (i.e., Asian) criteria for overweight $\left(\geq 23 \mathrm{~kg} / \mathrm{m}^{2}\right)$, obesity $\left(\geq 25 \mathrm{~kg} / \mathrm{m}^{2}\right)$, and WC ( $\geq 90 \mathrm{~cm}$ for men and $\geq 80 \mathrm{~cm}$ for women). Thus, further studies different criteria for obesity in other populations are needed.

With these caveats in mind, our findings indicate that hepatic steatosis and MD are significantly associated with increased CVD risk. The benefits of applying the composite term rather than NAFLD in the prediction of CVD risk include positive inclusion criteria and better discriminative ability in the stratification of individuals at different CVD risks, suggesting that the composite term needs to be considered an important risk factor for CVD.

\section{CONFLICTS OF INTEREST}

No potential conflict of interest relevant to this article was reported.

\section{ACKNOWLEDGEMENTS}

S.J. and S.M.K. received grants from the BK21-plus education program provided by the National Research Foundation of Korea.

\section{AUTHOR CONTRIBUTIONS}

Study concept and design: all authors. Data acquisition: S.J., S.C., J.C., S.M.K., J.S.S., G.L., W.K., S.M.P. Data analysis and interpretation: all authors. Drafting of the manuscript: S.J., W.K., S.M.P. Critical revision of the manuscript for important intellectual content: W.K., S.M.P. Statistical analysis: S.J., S.C. Study supervision: W.K., S.M.P.

\section{ORCID}

Seogsong Jeong https://orcid.org/0000-0003-4646-8998 Yun Hwan Oh https://orcid.org/0000-0002-1627-7528 Seulggie Choi https://orcid.org/0000-0002-0581-165X Jooyoung Chang https://orcid.org/0000-0002-8586-0645
Sung Min Kim

https://orcid.org/0000-0002-1957-2186

Joung Sik Son

https://orcid.org/0000-0003-2460-3378

Gyeongsil Lee

Won Kim

Sang Min Park

https://orcid.org/0000-0003-1910-9658

https://orcid.org/0000-0002-2926-1007

https://orcid.org/0000-0002-7498-4829

\section{SUPPLEMENTARY MATERIALS}

Supplementary materials can be accessed at https://doi. org/10.5009/gnl220256.

\section{REFERENCES}

1. Ludwig J, Viggiano TR, McGill DB, Oh BJ. Nonalcoholic steatohepatitis: Mayo Clinic experiences with a hitherto unnamed disease. Mayo Clin Proc 1980;55:434-438.

2. Younossi Z, Tacke F, Arrese M, et al. Global perspectives on nonalcoholic fatty liver disease and nonalcoholic steatohepatitis. Hepatology 2019;69:2672-2682.

3. Younossi Z, Anstee QM, Marietti M, et al. Global burden of NAFLD and NASH: trends, predictions, risk factors and prevention. Nat Rev Gastroenterol Hepatol 2018;15:11-20.

4. Eslam M, Sanyal AJ, George J; International Consensus Panel. MAFLD: a consensus-driven proposed nomenclature for metabolic associated fatty liver disease. Gastroenterology 2020;158:1999-2014

5. Eslam M, Newsome PN, Sarin SK, et al. A new definition for metabolic dysfunction-associated fatty liver disease: an international expert consensus statement. J Hepatol 2020;73:202209.

6. Stefan N, Schick F, Häring HU. Causes, characteristics, and consequences of metabolically unhealthy normal weight in humans. Cell Metab 2017;26:292-300.

7. Expert Panel on Detection, Evaluation, and Treatment of High Blood Cholesterol in Adults. Executive summary of the third report of the National Cholesterol Education Program (NCEP) Expert Panel on Detection, Evaluation, and Treatment of High Blood Cholesterol in Adults (Adult Treatment Panel III). JAMA 2001;285:2486-2497.

8. European Association for the Study of the Liver (EASL); European Association for the Study of Diabetes (EASD); European Association for the Study of Obesity (EASO). EASL-EASD-EASO Clinical Practice Guidelines for the management of non-alcoholic fatty liver disease. J Hepatol 2016;64:1388-1402.

9. Chalasani N, Younossi Z, Lavine JE, et al. The diagnosis and management of nonalcoholic fatty liver disease: practice guidance from the American Association for the Study of Liver Diseases. Hepatology 2018;67:328-357. 
10. Jeong S, Kim K, Chang J, et al. Development of a simple nonalcoholic fatty liver disease scoring system indicative of metabolic risks and insulin resistance. Ann Transl Med 2020;8:1414.

11. Kovalic AJ, Satapathy SK. The role of nonalcoholic fatty liver disease on cardiovascular manifestations and outcomes. Clin Liver Dis 2018;22:141-174.

12. Mottillo S, Filion KB, Genest J, et al. The metabolic syndrome and cardiovascular risk a systematic review and metaanalysis. J Am Coll Cardiol 2010;56:1113-1132.

13. Kwon S. Thirty years of national health insurance in South Korea: lessons for achieving universal health care coverage. Health Policy Plan 2009;24:63-71.

14. Seong SC, Kim YY, Park SK, et al. Cohort profile: the National Health Insurance Service-National Health Screening Cohort (NHIS-HEALS) in Korea. BMJ Open 2017;7:e016640.

15. Choi J, Kim HJ, Lee J, Cho S, Ko MJ, Lim YS. Risk of hepatocellular carcinoma in patients treated with entecavir vs tenofovir for chronic hepatitis B: a Korean nationwide cohort study. JAMA Oncol 2019;5:30-36.

16. Bedogni G, Bellentani S, Miglioli L, et al. The fatty liver index: a simple and accurate predictor of hepatic steatosis in the general population. BMC Gastroenterol 2006;6:33.

17. Kahn HS. The "lipid accumulation product" performs better than the body mass index for recognizing cardiovascular risk: a population-based comparison. BMC Cardiovasc Disord 2005;5:26.

18. Lee JH, Kim D, Kim HJ, et al. Hepatic steatosis index: a simple screening tool reflecting nonalcoholic fatty liver disease. Dig Liver Dis 2010;42:503-508.

19. Sundararajan V, Henderson T, Perry C, Muggivan A, Quan H, Ghali WA. New ICD-10 version of the Charlson comorbidity index predicted in-hospital mortality. J Clin Epidemiol 2004;57:1288-1294.

20. Writing Group Members, Mozaffarian D, Benjamin EJ, et al. Heart disease and stroke statistics: 2016 update. A report from the American Heart Association. Circulation 2016;133:e38-e360.

21. Son JS, Choi S, Kim K, et al. Association of blood pressure classification in Korean young adults according to the 2017
American College of Cardiology/American Heart Association Guidelines with subsequent cardiovascular disease events. JAMA 2018;320:1783-1792.

22. Eslam M, Sarin SK, Wong VW, et al. The Asian Pacific Association for the Study of the Liver clinical practice guidelines for the diagnosis and management of metabolic associated fatty liver disease. Hepatol Int 2020;14:889-919.

23. Kang SH, Cho Y, Jeong SW, Kim SU, Lee JW; Korean NAFLD Study Group. From nonalcoholic fatty liver disease to metabolic-associated fatty liver disease: big wave or ripple? Clin Mol Hepatol 2021;27:257-269.

24. Kim D, Kim WR, Kim HJ, Therneau TM. Association between noninvasive fibrosis markers and mortality among adults with nonalcoholic fatty liver disease in the United States. Hepatology 2013;57:1357-1365.

25. Lee H, Lee YH, Kim SU, Kim HC. Metabolic dysfunctionassociated fatty liver disease and incident cardiovascular disease risk: a nationwide cohort study. Clin Gastroenterol Hepatol 2021;19:2138-2147.

26. Targher G, Byrne CD, Lonardo A, Zoppini G, Barbui C. Non-alcoholic fatty liver disease and risk of incident cardiovascular disease: a meta-analysis. J Hepatol 2016;65:589-600.

27. Fernández-SolàJ. Cardiovascular risks and benefits of moderate and heavy alcohol consumption. Nat Rev Cardiol 2015;12:576-587.

28. Goel S, Sharma A, Garg A. Effect of alcohol consumption on cardiovascular health. Curr Cardiol Rep 2018;20:19.

29. Lo MK, Lee KF, Chan NN, et al. Effects of gender, Helicobacter pylori and hepatitis B virus serology status on cardiovascular and renal complications in Chinese type 2 diabetic patients with overt nephropathy. Diabetes Obes Metab 2004;6:223-230.

30. Petta S, Maida M, Macaluso FS, et al. Hepatitis C virus infection is associated with increased cardiovascular mortality: a meta-analysis of observational studies. Gastroenterology 2016;150:145-155.

31. Wang Y, Xiong J, Niu M, Xu W, Xu K, Zhong H. Hepatitis B virus and the risk of coronary heart disease: a comprehensive systematic review and meta-analyses of observational studies. Int J Cardiol 2018;265:204-209. 